\title{
Radiosensitization using hydrogen peroxide in patients with cervical cancer
}

\author{
RONG HU $^{1}$, ANNEYUKO I. SAITO ${ }^{1}$, TAIRA MITSUHASHI ${ }^{1}$, TATSUYA INOUE ${ }^{1}$, \\ TSUYOSHI OTA ${ }^{2}$, TAKAFUMI UJIHIRA ${ }^{2}$, KOYO YOSHIDA ${ }^{2}$ and KEISUKE SASAI ${ }^{1}$ \\ ${ }^{1}$ Department of Radiation Oncology, Juntendo University Faculty of Medicine, Bunkyo, Tokyo 113-8421; \\ ${ }^{2}$ Department of Obstetrics and Gynecology, Juntendo University Urayasu Hospital, Urayasu, Chiba 279-0021, Japan
}

Received October 16, 2020; Accepted March 9, 2021

DOI: $10.3892 / \mathrm{mco} .2021 .2304$

\begin{abstract}
The purpose of the present study was to analyze the feasibility and safety of radiosensitization using hydrogen peroxide for cervical cancer. In superficial tumors, breast cancer and hepatocellular carcinoma, the safety and effectiveness of radiosensitization has been reported; to the best of our knowledge, however, there are no reports on cervical cancer. A total of 20 patients with cervical cancer were recruited. Inclusion criteria were as follows: Patients who required radical external beam radiotherapy (RT); ineligible for or refused brachytherapy; age, $\geq 20$ years; no hematogenous metastasis; Eastern Cooperative Oncology Group Performance Status up to 2; and had not undergone prior treatment. Hydrogen peroxide was used twice a week in combination with RT. A $3 \%$ hydrogen peroxide solution-soaked gauze was inserted into the vagina during RT. A total of 45 Gy was delivered in 25 fractions to the whole pelvis with a boost of $10 \mathrm{~Gy}$ in 5 fractions if pelvic or para-aortic metastatic lymph nodes were observed. Ultimately, 18 patients were evaluated. Among the 17 patients (excluding one patient with tumor in situ), the oneand two-year overall survival rates were both $90 \%$ in patients with stage I/II and $86 \%$ in stage III/IV cervical cancer. The adverse events were well tolerated with no severe acute or late adverse events. Although limited by small sample size, short observation time and low radiation dose, the present study demonstrated that radiosensitization treatment may be an option for patients who cannot undergo brachytherapy. The
\end{abstract}

Correspondence to: Dr Anneyuko I Saito, Department of Radiation Oncology, Juntendo University Faculty of Medicine, 3-1-3 Hongo, Bunkyo, Tokyo 113-8421, Japan

E-mail: anyusaike@yahoo.co.jp

Abbreviations: RT, radiotherapy; ROS, reactive oxygen species; $\mathrm{O}_{2-}$, superoxide anion; HO, hydroxyl radical; LET, linear energy transfer; CT, computed tomography; LN, lymph node; CR, complete response; PD, progressive disease; SD, stable disease; OS, overall survival PFS, progression-free survival rate; EBRB, external-beam RT boost

Key words: radiosensitization, hydrogen peroxide, cervical cancer, tumor hypoxia study was retrospectively registered at the university hospital medical information network center (no. UMIN000039045) on January 6, 2020.

\section{Introduction}

Tumor hypoxia is a major constraint in the use of radiotherapy (RT) and numerous types of chemotherapy, such as alkylating agents, carboplatin and anthracyclines (1). Various pathogenetic mechanisms contribute to the development of hypoxia in solid tumors. Hypoxia is associated with malignant progression, increased tumor invasion, angiogenesis, and increased metastasis formation (2). Reactive oxygen species (ROS), including free radicals, such as superoxide anions $\left(\mathrm{O}_{2}-\right)$ and hydroxyl radicals (HO) and non-radical species such as $\mathrm{H}_{2} \mathrm{O}_{2}$, are effective molecules in RT, contributing to RT-induced DNA damage and cancer cell death. Enhancing ROS production by various means has been investigated as a radiosensitizing strategy (3). Ogawa (4) studied the effect of tumors irradiated in the presence of hydrogen peroxide and found that the activity of anti-oxidative enzymes, such as peroxidase and catalase, were blocked while oxygen molecules were simultaneously produced via the $\mathrm{H}_{2} \mathrm{O}_{2}$ effect, in which $\mathrm{H}_{2} \mathrm{O}_{2}$ produced by reactive oxygen species accumulates in the cytoplasm and then moves into the lysosomes, where it causes lysosomal membrane dysfunction and ultimately apoptosis, resulting in oxidative damage to low-linear energy transfer (LET)-radioresistant tumor cells, thereby rendering them highly sensitive to irradiation. UK reseachers to aimed to confirm the safey and efficacy of Kochi Oxydol Radiation Therapy for Unresectable Carcinomas (KORTUC) in breast cancer. Nimalasena et al (5) conducted a Phase I clincal trial, which involved 12 patients with locally advanced breast cancer, and demonstrated the safety and tolerability of intravaginal $\mathrm{H}_{2} \mathrm{O}_{2}+$ external beam RT; a Phase II control arm trial by the same UK group is starting now (6).

There were 604,127 new cervical cancer cases reported worldwide in 2020, which made cervical cancer the fourth most common cancer among women globally (7). External RT combined with brachytherapy and chemotherapy is the standard treatment for cervical cancer (8). Brachytherapy involves the application of a radioactive source in close proximity to the tumor. It takes advantage of the inverse-square law whereby 
the RT dose is inversely proportional to the square of the distance from the source, allowing for a high dose to the tumor with relative sparing of the surrounding normal structure. Brachytherapy is the only demonstrated method of providing a high dose required to control cervical cancer without causing severe side effects (9).

In Japan, the delivery of treatment to patients with cervical cancer is complicated by various obstacles. Among 897 radiation oncology facilities, only $163(18 \%)$ are equipped with brachytherapy; of those, only 150 (17\% of all facilities) have a machine in use (10). Moreover, because brachytherapy procedures are uncomfortable, certain patients refuse this treatment. In order to address this problem and in light of prior work by Ogawa et al $(11,16)$, the present study sought to analyze the feasibility and safety of radiosensitization using hydrogen peroxide as a substitute for brachytherapy for the treatment of cervical cancer.

\section{Materials and methods}

Under the Juntendo Urayasu Hospital's review board approval, patients with cervical caner requiring radiosensitization treatment due to an inability to undergo standard therapy were recruited from Juntendo Urayasu Hospital between February 2014 and August 2019. Eligible patients had cervical cancer of any pathology; required RT; were ineligible for or refused brachytherapy; aged $\geq 20$ years; were without hematogenous metastasis; had an Eastern Cooperative Oncology Group Performance Status up to 2; and had not undergone prior treatment. No restrictions were imposed regarding the use or type of concomitant chemotherapy. Patients who met the study criteria were examined, the therapy was explained and, for the patients who refused brachytherpy, it was emphasized that brachytehrapy was the most suitable method and that the survival rates of using external irradiation alone are worse than those of combination brachytherapy. After ensuring full understanding and obtaining written informed consent, participants were enrolled in the study. Treatment planning was performed on a Pinnacle 3 treatment planning system (Philips Medicals Systems, Inc.) with computed tomography (CT) imaging (GE High Speed; GE Healthcare Japan). Patients began treatment two working days after CT imaging. The clinical target volume was created by contouring the uterus, proximal vagina, paracervical and parametrial tissue, including uterosacral ligaments, and pelvic nodal basins. If positive lymph nodes (LN) were detected in the common iliac node or fare above, para-aortic nodal basins were also contoured. The planning target volume incorporated an additional $5 \mathrm{~mm}$ set up margin to the clinical target volume.

Hydrogen peroxide was used twice per week (Monday and Thursday or Tuesday and Friday) in combination with RT. Radiosensitization treatment was performed only twice per week because mucous membranes are more sensitive to X-rays than skin (17), so a relatively low frequency was selected to avoid severe adverse events. Immediately before RT, a gauze soaked in $3 \%$ hydrogen peroxide solution was inserted into the vagina, ensuring firm contact with the lesion. The gauze was removed immediately following RT. The gauze was intended to be inserted only for the duration of treatment ( 10 min). However, because the Gynecology Department is $10 \mathrm{~min}$ walk from the Department of Radiation Oncology, the gauze typically remained inserted for 20-30 $\mathrm{min}$. The procedure was performed by the attending gynecologist. The RT dose was 45 Gy over 25 fractions delivered to the whole pelvis from four directions using 10-MV X-rays from a linear accelerator (Elekta Synergy Platform; Elekta Instrument AB) five days per week. For pelvic metastatic LN, a boost of $10 \mathrm{~Gy}$ was delivered in 5 fractions. The moderate total RT dose was selected in consideration of potential salvage surgery in case of residual tumor following RT. In case of metastatic para-aortic LN but absent distant metastases, the para-aoritic area was included within the first treatment field and a 10 Gy boost was delivered.

The primary objective was to assess the safety and toxic effects of radiosensitization with hydrogen peroxide. The dose limiting toxicity was grade three or worse vaginal mucositis. Secondary objectives for the present study included locoregional control after one month, progression-free survival (PFS) and overall survival (OS).

Acute and late adverse events were evaluated at one and six months after completion of RT, as per the Common Terminology Criteria for Adverse Events, Version 4.0 (18). Efficacy of the treatment was evaluated in reference to Response Evaluation Criteria in Solid Tumours (RECIST) (19). In brief, complete response (CR) was defined as disappearance of target lesions, partial response was defined as $\geq 30 \%$ decrease in the sum of diameters of target lesions and progressive disease (PD) was definced as $\geq 20 \%$ increase in the sum of diameters of target lesions. Any other response was defined as stable disease (SD). Evaluation was performed by comparing CT and magnetic resonance images and pelvic examination findings before and 1-3 months after completion of RT.

\section{Results}

Reasons for selecting radiosensitization treatment over brachytherapy included severe congenital hip joint dislocation precluding the use of brachytherapy $(n=2)$, huge tumor size $(n=1)$ and refusal of brachytherapy $(n=15)$. Because there is no brachytherapy facility within Juntendo University Urayasu Hospital, patients must travel $>1$ h to the nearest hospital to undergo brachytherapy. Since adverse events due to chemotherapy and RT are expected (20), many patients experience anxiety about such travel and refuse brachytherapy.

A total of 20 patients met the inclusion criteria. Of these, one received only once-per-week gauze insertion due to a protocol deviation. One patient discontinued treatment after two radiosensitization treatments due to overall poor health and died soon after. This was a 78-year-old patient with small cell cancer. At admission, she exhibited para-aortic LN metastasis (stage IVB) but was near full ambulatory (performance status 1). However, tumor progression was rapid and on the second day of radiation she developed bilateral hydronephrosis. On the third day of radiation, a percutaneous nephrostomy was placed in the bilateral kidney. Over the weekend, she developed a high fever due to a urinary tract infection. On Monday (day 5 of radiation), her blood pressure dropped; gauze insertion was stopped but irradiation was continued to decrease the size of the tumor. Radiation was stopped on day 7 because she was in critical condition. She died nine days later. This patient had received gauze insertion twice and 7 fractions of 1.8 Gy 
to the whole pelvis and para-aorta. One patient switched to brachytherapy following three radiosensitization treatments owing to a change of mind. Two patients who discontinuted treatment were excluded. Regarding concurrent chemotherapy, 14 patients received cisplatin, two received 5-fluorouracil and two underwent no chemotherapy (one of whom had early stage disease, while the other had kidney dysfuction).

As a result, 18 patients were included in the analysis (mean age, 64.5 years; range, 37-83 years). The stage distribution was as follows: Tumor in situ, 1; stage IB, 2; IIA, 2; IIB, 6; IIIB, 2 and IVB, 5. Of these 18 patients, 4 had pelvic LN metastases (22\%) and 3 had both pelvic and paraaortic LN metastases (17\%). Patient characteristics are summarized in Table I. Treatments are summarized in Tables II and III provides a summary of individual patient outcomes.

Outcomes were evaluated one month after completion of treatment. Overall, 15 patients achieved CR (83\%) and three achieved PR (17\%). Of those achieving CR, five experienced pelvic recurrence at $6,12,13,16$ and 18 months after completing RT (of these, three had pelvic LN metastases before treatment); all three patients who achieved PR exhibited disease progression and mortality due to distant metastases or severe cachexia at 4, 8 and 23 months after RT completion (of these, one had pelvic metastasis before treatment). The remaining ten maintained CR at the time of analysis. The one- and two-year PFS was 69.0 and $55.2 \%$ and the one- and two- year OS was 81.6 and $68.0 \%$, respectively.

Among the five CR patients who experienced local (pelvic) recurrence, cervical tumor size was $>50 \mathrm{~mm}$ (mean maximum diameter, $62 \mathrm{~mm}$ ). In comparison, of the ten CR patients who showed no sign of recurrence by the end of the study, only four had tumors $>50 \mathrm{~mm}$ in size (mean maximum diameter, $44 \mathrm{~mm}$; Table III).

The acute and late adverse events are summarized in Table IV. The adverse events included diarrhea, mucositis, nausea, neutropenia, anemia and thrombocytopenia. Treatment was well tolerated with no acute Grade 3 or worse mucositis due to gauze insertion. A total of 15 patients whose follow-up exceeded 6 months,including one who died at 10 months, (median, 28 months) were evaluated for late adverse events. No notable late adverse events had been observed by the end of analysis.

\section{Discussion}

In 2008, to improve the effect of low-LET RT, Ogawa et al (11) developed a novel radiosensitization treatment called KORTUC, which uses a $3 \% \mathrm{w} / \mathrm{v}$ hydrogen peroxide solution-soaked gauze for superficially exposed and unresectable neoplasms, such as malignant melanoma and malignant fibrous histiocytoma. They analyzed five patients who received 48 Gy over 12 fractions three times per week. Two patients showed CR and the remaining three experienced PR without severe complications. In 2011, Ogawa et al developed a novel radiosensitizer for intratumoral injection called KORTUC II, comprising a combination of hydrogen peroxide and sodium hyaluronate. A total of 52 patients with unresectable or recurrent neoplasms were enrolled and followed for at least a year. No patients experienced severe adverse effects. RECIST-determined CR and PR rates were 57 and 26\%, respectively and one-year survival
Table I. Patient $(n=18)$ and tumor characteristics.

Characteristic

Number of patients

\begin{tabular}{lc}
\hline Pathology & \\
Squamous cell carcinoma & 14 \\
Adenocarcinoma & 2 \\
Adenosquamous carcinoma & 2 \\
Stage & \\
I & 2 \\
II & 8 \\
III & 2 \\
IV & 5 \\
Tumor in situ & 1 \\
Performance status & \\
0 & 17 \\
1 & 1 \\
\hline
\end{tabular}

Table II. Treatment administered to patients $(n=18)$.

\begin{tabular}{lr} 
Treatment method & Number of \\
\hline Pelvic irradiation only & 10 \\
Boost to LN only & 4 \\
Para-aortic LN irradiation only & 0 \\
Boost + para-aotic LN irradiation & 4 \\
Concomitant chemotherapy & \\
Cisplatin & 14 \\
5-fluorouracil & 2 \\
No chemotherapy & 2
\end{tabular}

LN, lymph node.

was $74 \%$ (12). In 2016, Aoyama et al (13) reported a follow-up study of 20 patients with recurrent breast cancer from the aforementioned cohort. The total dose was 44.00-49.50 Gy (X-ray irradiation) or 40.00-48.00 Gy (electron beam irradiation). Of the 24 lesions presented by the 20 patients, 18 exhibited CR, 5 exhibited PR, 0 was SD and 1 was PD. The one- and two-year OS rates were 100 and 95\%, respectively. In 2017, Aoyama et al (14) reported a follow up study of seven patients with unresectable breast cancer from the aforementioned cohort. The total RT dose was 44.0-49.5 Gy over 16-18 fractions. Injection was initiated from the sixth RT fraction and was performed twice per week. The OS was 100 and $86 \%$ at one and two years post-treatment, respectively.

In 2014, treatment with an injection of hydrogen peroxide and sodium hyaluronate into a tumor immediately prior to intraoperative RT was tested in patients with stage IVA locally advanced unresectable pancreatic cancer followed by externalbeam RT and systemic chemotherapy (15). The one- and two-year survival rates for the 12 patients in this analysis were 75 and $25 \%$, respectively. There were no serious complications. A retrospective study (16) investigated 72 patients with stage I-II breast cancer who received 
Table III. Summary of individual patient outcomes.

\begin{tabular}{|c|c|c|c|c|c|c|c|c|}
\hline Patient & $\begin{array}{l}\text { Age, } \\
\text { years }\end{array}$ & Stage & Pathology & $\begin{array}{c}\text { Mean } \\
\text { maximun } \\
\text { diameter, } \mathrm{mm}\end{array}$ & Outcome & $\begin{array}{c}\text { Follow-up } \\
\text { period, months }\end{array}$ & $\begin{array}{l}\text { Recurrence } \\
\text { period, } \\
\text { months }\end{array}$ & Recurrence site \\
\hline 1 & 80 & Tis & SCC & 0 & $\mathrm{CR}$ & 25 & N/A & N/A \\
\hline 2 & 72 & IB & $\mathrm{SCC}$ & 33 & $\mathrm{CR}$ & 32 & N/A & N/A \\
\hline 3 & 37 & IB & SCC & 40 & $\mathrm{CR}$ & 41 & N/A & N/A \\
\hline 4 & 83 & IIA & SCC & 27 & $\mathrm{CR}$ & 57 & N/A & N/A \\
\hline 5 & 75 & IIA & SCC & 70 & $\mathrm{CR}$ & 48 & N/A & N/A \\
\hline 6 & 71 & IIB & $\mathrm{ADE}$ & 55 & $\begin{array}{l}\mathrm{PR} \rightarrow \mathrm{PD} \\
\text { (mortality) }\end{array}$ & 10 (mortality) & 4 & Lung \\
\hline 7 & 54 & IIB & SCC & 60 & $\mathrm{CR}$ & 32 & N/A & N/A \\
\hline 8 & 58 & IIB & SCC & 50 & $\mathrm{CR} \rightarrow$ Recurrence & 25 (mortality) & 18 & Vagina \\
\hline 9 & 40 & IIB & SCC & 78 & $\mathrm{PR} \rightarrow \mathrm{PD}$ & 24 (mortality) & 1 & Iliac LN \\
\hline 10 & 70 & IIB & SCC & 50 & $\mathrm{CR} \rightarrow$ Recurrence & 25 & 6 & Iliac LN and sacral bone \\
\hline 11 & 64 & IIB & SCC & 80 & $\mathrm{CR}$ & 24 & N/A & N/A \\
\hline 12 & 65 & IIIB & SCC & 60 & $\mathrm{CR} \rightarrow$ Recurrence & 26 & 12 & Bladder \\
\hline 13 & 66 & IIIB & SCC & 42 & $\mathrm{CR}$ & 24 & N/A & N/A \\
\hline 14 & 62 & IVA & SCC & 51 & $\mathrm{CR} \rightarrow$ Recurrence & 31 & 13 & Cervix \\
\hline 15 & 68 & IVA & $\mathrm{SCC}$ & 52 & $\mathrm{CR} \rightarrow$ Recurrence & 30 & 16 & Groin LN \\
\hline 16 & 42 & IVA & ADSQ & 75 & $\mathrm{PR} \rightarrow \mathrm{PD}$ & 5 (mortality) & 3 & $\begin{array}{l}\text { Peritoneum } \\
\text { and pelvic LN }\end{array}$ \\
\hline 17 & 44 & IVA & $\mathrm{ADE}$ & 40 & $\mathrm{CR}$ & 33 & N/A & $\mathrm{N} / \mathrm{A}$ \\
\hline 18 & 54 & IVA & ADSQ & 30 & $\mathrm{CR}$ & 24 & N/A & N/A \\
\hline
\end{tabular}

Patient 4 only once-per-week gauze insertion due to a protocol mistake. Tis, tumor in situ; SCC, squamous cell carcinoma; ADE, adenocarcinoma; ADSQ, adenosquamous carcinoma; CR, complete response; PR, partial response; PD, progressive disease; N/A, not applicable; LN, lymph node.

KORTUC II treatment between 2006 and 2014. The total RT dose was 44 Gy over 16 fractions followed by electron boost of 3 Gy three times. The 5-year OS, PFS and local control rates were $100.0,97.1$ and $97.1 \%$, respectively.

External-beam RT combined with brachytherapy and chemotherapy is the standard treatment for stage IB2-IVA cervical cancer (8). In Japan, because of the lack of brachytherapy facilities (10), patients must travel to a remote hospital during RT or forego brachytherapy altogether. The situation is particulary challenging in resource-limited countries: It has been reported that only 20 of 52 African countries provided brachytherapy in 2010 and only nine centers in Latin America perform gynecological brachytherapy (21).

In order to combat this problem, the present prospective study analyzed the feasibility and safety of radiosensitization treatment using hydrogen peroxide for patients with cervical cancer who cannot undergo brachytherapy. In order to be considered as a suitable option for patients who are unsuitable for brachytherapy, radiosensitization treatment must be proved to be non-inferior compared with standard treatment, including brachytherapy. Yang et al (22) analysed the Surveillence, Epidemiology, and End Results database to compare the prognostic impact of several treatment modalities, including RT with or without brachytherapy, for cervical cancer. They showed that external-beam RT alone was, in most cases, less effective than combined external-beam RT and brachytherapy.
Quinn et al (23) reported the results of a multi-institutional international retrospective survival analysis of 15,081 patients. Among them, the 1,655 patients who received RT with brachytherapy had 1- and 2-year survival rates as follows: Stage IB1, 95.8 and 85.2; IIA, 94.0 and 83.7; IIB, 92.2 and 81.5 and IVA, 74.0 and $48.6 \%$. Corresponding rates in the present study were 100 and 100 (4/4) for stage IB1 and IIA, 83 and 83 (5/6) for IIB, 100 and 100 (2/2) for IIIB and 80 and 80\% (4/5) for IVA. Since the patient population was small, the reliability of this comparison is limited, but it is encouraging that the present results were not notably worse than standard treatment. Therefore, this method may be an option for patients who are unsuitable for or unable to undergo brachytherapy.

Retrospective reports regarding patients with cervical cancer who did not undergo brachytherapy and received only external-beam RT are summarized in Table V (24-29). The one- and two-year OS was 74-100 and 43-64\%, which was comparable to the present study. Regarding late toxicity, studies including total dose $>70$ Gy $(24,25)$ or accelerated hyperfractionation (26), the number of patients with Grade 2 or worse late toxicity was $10-23.7 \%$. On the other hand, studies with conventional fractionation where the dose did not exceed $70 \mathrm{~Gy}$, late toxicity was $0.7-9.1 \%$. In the present study there was no late toxicity; the total radiation dose used was $45 \mathrm{~Gy}$. In the aforementioned studies, no patients received a total dose $<55$ Gy. 
Table IV. Adverse events assessed according to Common Terminology Criteria for Adverse Events (version 4.0).

\begin{tabular}{lcccc}
\hline & \multicolumn{3}{c}{ Grade } \\
\cline { 2 - 5 } Adverse event & 0 & 1 & 2 & 3 \\
\hline Mucositis & $8(47.1 \%)$ & $8(47.1 \%)$ & $1(5.9 \%)$ & 0 \\
Diarrhea & $2(11.8 \%)$ & $10(58.8 \%)$ & $4(23.5 \%)$ & $1(5.9 \%)$ \\
Cystitis & 0 & 0 & 0 & 0 \\
Neutropenia & $7(41.2 \%)$ & $2(11.8 \%)$ & $4(23.5 \%)$ & 0 \\
Anemia & $1(5.9 \%)$ & $8(47.1 \%)$ & $4(23.5 \%)$ & $3(17.7 \%)$ \\
Thrombocytopenia & $9(52.9 \%)$ & $5(29.4 \%)$ & $2(11.8 \%)$ & 0 \\
Nausea & $10(58.8 \%)$ & $6(35.3 \%)$ & $1(5.9 \%)$ & 0 \\
Late & 0 & 0 & 0 & 0 \\
\hline
\end{tabular}

All observed adverse events were acute; no late adverse events were observed.

Table V. Retrospective reports of patients with cervical cancer who were unable to recieve brachytherapy and received externalbeam radiotherapy alone.

\begin{tabular}{|c|c|c|c|c|c|c|}
\hline Author, year & $\begin{array}{l}\text { Number } \\
\text { of patients }\end{array}$ & Stage & Treatment & PFS & OS & Late toxicity \\
\hline The present study & $17^{\mathrm{a}}$ & IB-IVA $^{\mathrm{a}}$ & Pelvis, $45 \mathrm{~Gy} / 25 \mathrm{fx}$ & $\begin{array}{l}1 \text { year, } 69.0 \% \\
2 \text { year, } 55.2 \%\end{array}$ & $\begin{array}{l}1 \text { year, } 81.6 \% \\
2 \text { year, } 68.0 \%\end{array}$ & $\begin{array}{l}\text { GI, } 0 \% \text { (any); } \\
\text { GU, } 0 \% \text { (any) }\end{array}$ \\
\hline $\begin{array}{l}\text { Karlsson et al, } \\
2017 \text { (24) }\end{array}$ & 86 & I-IV & $\begin{array}{l}\text { Pelvis, 46-68 Gy/23-34 fx; } \\
\text { Tumor boost, 6-26 Gy/3-13 fx }\end{array}$ & NR & $\begin{array}{l}1 \text { year, } 70.0 \%^{\mathrm{b}} \\
2 \text { year, } 50.0 \%^{\mathrm{b}}\end{array}$ & $\begin{array}{c}\text { GI, } 23.7 \% \\
\text { (grade } 2-4 \text { ); GU, } \\
10.5 \% \\
\text { (grade } 2-4)\end{array}$ \\
\hline $\begin{array}{l}\text { Barraclough } \\
\text { et al, } 2008(25)\end{array}$ & 44 & IB-IVA & $\begin{array}{c}\text { Pelvis, } 40-45 \mathrm{~Gy} / 25 \mathrm{fx} \\
\text { Tumor boost, } 15-25 \\
\text { Gy/8-10 fx }\end{array}$ & NR & $\begin{array}{l}1 \text { year, } 81.4 \% \\
2 \text { year, } 64.0 \%\end{array}$ & $\begin{array}{c}\text { GI, } 9.1 \% \\
\text { (grade } 2) ; \mathrm{GU}, \\
2.3 \%(\text { grade } 3)\end{array}$ \\
\hline $\begin{array}{l}\text { Matsuura et al, } \\
2012(26)\end{array}$ & 16 & IIB-IVA & $\begin{array}{l}\text { Pelvis, } 45 \mathrm{~Gy} / 25 \mathrm{fx} \text {; } \\
\text { Tumor boost }(\mathrm{AHF}) \text {, } \\
\text { 9-15 Gy/6-10 fx }\end{array}$ & NR & 3 year, $43.8 \%$ & $\begin{array}{c}\text { GI, } 12.5 \% \\
\text { (grade } 2-4) ; \mathrm{GU}, \\
12.5 \% \text { (grade } 2)\end{array}$ \\
\hline Ito et al, 2019 (27) & 37 & IB-IVA & $\begin{array}{c}\text { Pelvis, } 45-50 \mathrm{~Gy} / 25 \mathrm{fx} \\
\text { Tumor boost, } 6-10 \\
\text { Gy/3-5 fx }\end{array}$ & $\begin{array}{l}1 \text { year, } 45.0 \% \\
2 \text { year, } 29.0 \%\end{array}$ & $\begin{array}{l}1 \text { year, } 74.0 \% \\
2 \text { year, } 43.0 \%\end{array}$ & $\begin{array}{l}\mathrm{GU}, 2.7 \% \\
\text { (grade } 4) ; \mathrm{GU}, \\
2.7 \%(\text { grade } 4)\end{array}$ \\
\hline $\begin{array}{l}\text { Park et al, } \\
2010(28)\end{array}$ & 10 & IIA-IIIB & $\begin{array}{c}\text { Pelvis, } 40-50 \\
\text { Gy/20-25 fx; } \\
\text { Tumor boost, } 25-30 \\
\text { Gy/6-12 fx }\end{array}$ & NR & 1 year, $100 \%$ & $\begin{array}{c}\text { GI, } 10.0 \% \\
\text { (grade 2); } \\
\text { Subcutaneous } \\
\text { abscess, } 10.0 \%\end{array}$ \\
\hline $\begin{array}{l}\text { Saibishkumar et al, } \\
2006(29)\end{array}$ & 146 & I -IVA & $\begin{array}{c}\text { Pelvis, } 46 \mathrm{~Gy} / 23 \mathrm{fx} \\
\text { Tumor boost, } 14-20 \\
\text { Gy/7-10 fx }\end{array}$ & 5 year, $11.6 \%$ & 5 year, $15.1 \%$ & $\begin{array}{c}\text { GI, } 0.7 \% \\
\text { (grade 3); GU, } \\
0.7 \% \text { (grade 3) }\end{array}$ \\
\hline
\end{tabular}

${ }^{\mathrm{a} E x c l u d i n g}$ patient with tumor in situ. ${ }^{\mathrm{b} C a n c e r-s p e c i f i c ~ s u r v i v a l . ~ P F S, ~ p r o g r e s s i o n-f r e e ~ s u r v i v a l ; ~ O S, ~ o v e r a l l ~ s u r v i a l ; ~ G y, ~ g r a y ; ~ f x, ~ f r a c t i o n ; ~}$ GI, gastrointestinal; GU, genitourinary; AHF, accelerated hyperfractionation; NR, not reported.

Univariate and multivariate by Ito et al (27) showed that maximum primary tumor diameter $>5 \mathrm{~cm}$ is associated with significantly worse PFS.

Karlsson et al (24) compared the outcomes of patients underoing RT with brachytherapy with those of patients receiving external-beam RT boost (EBRB) instead of brachytherapy; the brachytherapy group fared better overall. The cancer-specific survival at one- and two-year was $~ 95$ and $85 \%$ for the brachytherapy group and 70 and $50 \%$ for the EBRB group. However, in patients with International Federation of 
Gynecology and Obstetrics stages III-IV, there was no significant difference in cancer-specific survival rate between the two groups.

Yoshida et al (30) reported that, for patients with tumors $>5 \mathrm{~cm}$, satistfactory outcomes were achieved by high quality image-guided adaptive brachytherapy with interstitial brachytherapy. Similar results was observed in the present study, where the one- and two- year local control rates were $100(17 / 17)$ and 93\% (14/15), respectively and the one- and two- year CR rates for all stages combined were 82 (14/17) and 59\% (10/17), respectively. In the present study all five patients with local recurrence had a tumor diameter $>5 \mathrm{~cm}$, whereas among the six patients with stage IIB cancer, only two achieved CR. By contrast, the two patients with stage IIA cancer both experienced CR without recurrence for more than four years. It may be that, similar to brachytherapy, tumor size is associated with the treatment outcome. Therefore, patients with smaller cervical tumors that do not develop toward the pelvic wall may benefit from radiosensitization treatment. However, patients who are not candidates for traditional brachytherapy but have larger tumors or those with advanced stage lesions may fare better with EBRB (24).

The present study is not without limitation. First, as aforementioned, the patient population was small and the observation time was relatively short. Furthermore, the radiation dose was relatively low in consideration of potential salvage surgery in case of residual tumor following RT. One of the present patients underwent salvage surgery.

Hong et al (31) reported that salvage surgery performed for cervical cancer following definitive RT with doses up to $54 \mathrm{~Gy}$ does not cause severe complications and achieves long-term survival. Therefore, increasing the dose or delivering additional EBRB to the uterus and cervix may improve results. Additionally, Ogawa et al (11) reported the safety of hydrogen peroxide solution-soaked gauze bolus on the skin during each RT session; however, in the present study, patients received KORTUC to the vaginal mucosa during only 2 of the 5 weekly RT sessions. Based on our safety results (Table IV), more frequent use of a vaginally inserted hydrogen peroxide solution-soaked gauze may improve outcomes.

In the present study, radiosensitization treatment using hydrogen peroxide appeared to be safe and feasible in patients with cervical cancer. This treatment could be a promising option for patients with small tumors that do not extend toward the pelvic wall who cannot undergo brachytherapy.

Future research, including clinical trials, is warranted to determine the efficacy of this treatment. For much of the prior experience is with injected KORTUC, theoretically, injection of KORTUC dirctly into the tumor may extend its benefits into deep tumor tissues. Future studies should investigate the efficacy of injection of KORTUC in patients with cervical cancer during RT.

Radiosensitization with gauze insertion before each radiation dose may improve treatment outcome. A synergistic effect may be achieved by combining KORTUC and brachytherapy. Moreover, increasing the radiation dose or delivering additional EBRB to the uterus and cervix might improve results. KORTUC is a promising method and future research should investigate how to maximize patient benefit.

\section{Acknowledgements}

The authors would like to thank Dr Robert Kneller (University of Tokyo, Japan) for helpful comments.

\section{Funding}

No funding was received.

\section{Availability of data and materials}

The datasets used and/or analyzed during the current study are available from the corresponding author on reasonable request.

\section{Authors' contributions}

AIS, TM, TI, TO, TU and KY obtained the data. RH and AIS conceptualized and designed the study, analyzed and interpreted data and drafted the manuscript. AIS and RH confirm the authenticity of all the raw data. KS was involved in the concept and design of the study. All authors revised the manuscript critically for important intellectual content and agreed to be accountable for all aspects of the work in ensuring that questions related to the accuracy or integrity of any part of the work are appropriately investigated and resolved. All authors read and approved the final manuscript.

\section{Ethics approval and consent to participate}

The present study was approved by the Institutional Review Board of Juntendo University Urayasu Hospital (approval no. 2014-008). Informed consent was obtained from all patients.

\section{Patient consent for publication}

Written informed consent for publication was obtained from all patients enrolled in the present study.

\section{Competing interests}

The authors declare that they have no competing interests.

\section{References}

1. Teicher BA: Hypoxia and drug resistance. Cancer Metastasis Rev 13: 139-168, 1994.

2. Weinmann M, Belka C and Plasswilm L: Tumor hypoxia: Impact on biology, prognosis and treatment of solid malignant tumors. Oncology 27: 83-90, 2004.

3. Wang $\mathrm{H}$, Jiang $\mathrm{H}$, Van De Gucht $\mathbf{M}$ and De Ridder $\mathbf{M}$ : Hypoxic Radioresistance: Can ROS be the key to overcome it? Cancers (Basel) 11: 112, 2019.

4. Ogawa Y: Paradigm shift in radiation biology/radiation oncology-exploitation of the ' $\mathrm{H}_{2} \mathrm{O}_{2}$ Effect' for radiotherapy using low-LET (Linear Energy Transfer) radiation such as X-rays and high-energy electrons. Cancers (Basel) 8: 28, 2016.

5. Nimalasena S, Gothard L, Anbalagan S, Allen S, Sinnett V, Mohammed K, Kothari G, Musallam A, Lucy C, Yu S, et al: Intratumoural hydrogen peroxide with radiotherapy in locally advanced breast cancer: Results from a phase I clinical trial. Int J Radiat Oncol Biol Phys 108: 1019-1029, 2020.

6. KORTUC Phase II - Intra-tumoural radiation sensitizer in patients with locally advanced/recurrent breast cancer. https://clinicaltrials. gov/ct2/show/NCT03946202. Accessed September 12, 2020. 
7. Cervix Uteri.GlobalCancerObservatory.https://gco.iarc.fr/today/ data/factsheets/cancers/23-Cervix-uteri-fact-sheet.pdf. Accessed March 12021.

8. CERV-6.NCCNGuidelines Version 1.2021CervicalCancerNCCN Evidence Blocks ${ }^{\mathrm{TM}}$. https://www.nccn.org/professionals/ physician_gls/pdf/cervical_blocks.pdf. Accessed March 1, 2021.

9. Banerjee R and Kamrava M: Brachytherapy in the treatment of cervical cancer: A review. Int J Womens Health 6: 555-564, 2014

10. Japanese Structure Survey of Radiation Oncology in 2015 (First Report). https://www.jastro.or.jp/medicalpersonnel/data center/cat6/cat/2015.html. Accessed 12 September 2020.

11. Ogawa Y, Ue H, Tsuzuki K, Tadokoro M, Miyatake K, Sasaki T, Yokota N, Hamada N, Kariya S, Hitomi J, et al: New radiosensitization treatment (KORTUC I) using hydrogen peroxide solution-soaked gauze bolus for unresectable and superficially exposed neoplasms. Oncol Rep 19: 1389-1394, 2008.

12. Ogawa Y, Kubota K, Ue H, Tadokoro M, Matsui R, Yamanishi T, Hamada N, Kariya S, Nishioka A, Nakajima H, et al: Safety and effectiveness of a new enzyme-targeting radiosensitization treatment (KORTUC II) for intratumoral injection for low-LET radioresistant tumors. Int J Oncol 39: 553-560, 2011.

13. Aoyama N, Ogawa Y, Yasuoka M, Takahasi M, Iawasa H, Miyatake K, Yamanishi T,Hamada N,TamuraT,KobayashiK, etal: Therapeutic response to a novel enzyme-targeting radiosensitization treatment (Kochi oxydol-radiation therapy for unresectable carcinomas) in patients with recurrent breast cancer. Oncol Lett 12: 29-34, 2016.

14. Aoyama N, Ogawa Y, Yasuoka M, Iwasa H, Miyatake K, Yoshimatsu R, Yamanishi T, Hamada N, Tamura T, Kobayashi K, et al: Therapeutic response to a novel enzymetargeting radiosensitization treatment (KORTUC II) for residual lesions in patients with stage IV primary breast cancer, following induction chemotherapy with epirubicin and cyclophosphamide or taxane. Oncol Lett 13: 69-76, 2017.

15. Nishioka A, Ogawa Y, Miyatake K, Tadokoro M, Nogami M, Hamada N, Kubota K, Kariya S, Kohsaki T, Saibara T, et al: Safety and efficacy of imageguided enzyme-targeting radiosensitization and intraoperative radiotherapy for locally advanced unresectable pancreatic cancer. Oncol Lett 8: 404-408, 2014.

16. Ogawa Y, Kubota K, Aoyama N, Yamanishi T, Kariya S, Hamada N, Nogami M, Nishioka A, Onogawa M and Miyamura $M$ : Non-surgical breast-conserving treatment (KORTUC-BCT) using a new radiosensitization method (KORTUC II) for patients with stage I or II breast cancer. Cancers (Basel) 7: 2277-2289, 2015.

17. 3.2Mechanism of Causing Effects on Human Body. BOOKLET to Provide Basic Information Regarding Health Effects of Radiation (1st edition). Ministry of the Enviroment Government of Japan. https://www.env.go.jp/en/chemi/rhm/basic-info/1st/ Assecced March 3, 2021.

18. Common Terminology Criteria for Adverse Events (CTCAE) Version 4.0. National cancer institute. https://ctep.cancer.gov/ protocolDevelopment/electronic_applications/ctc.htm\#ctc_50. Accessed September 12, 2020.
19. Eisenhauer EA, Therasse P, Bogaerts J, Schawartz LH, Sargent D, Ford R, Dancey J, Arbuck S, Gwyther S, Mooney M, et al: New response evaluation criteria in solid tumours: Revised RECIST guideline (version 1.1). Eur J Cancer 45: 228-247, 2009.

20. CERV-G.NCCNGuidelines Version 1.2021CervicalCancerNCCN Evidence BlocksTM. https://www.nccn.org/professionals/ physician_gls/pdf/cervical_blocks.pdf. Accessed March 1, 2021.

21. Small W Jr, Bacon MA, Bajaj A, Chuang LT, Fisher BJ, Harkenrider MM, Jhingran A, Kitchener HC, Mileshikin LR, Viswanathan AN and Gaffney DK: Cervical cancer: A global health crisis. Cancer 123: 2404-2412, 2017.

22. Yang J, Cai H, Xiao ZX, Wang H and Yang P: Effect of radiotherapy on the survival of cervical cancer patients: An analysis based on SEER database. Medicine (Baltimore) 98: e16421, 2019.

23. Quinn MA, Benedet JL, Odicino F, Maisonneuve P, Beller U, Creasman WT, Heintz AP, Nagan HY and Pecorelli S: Carcinoma of the cervix uteri. FIGO 26th annual report on the results of treatment in gynecological cancer. Int J Gynaecol Obstet 95 (Suppl 1): S43-S103, 2006.

24. Karlsson J, Dreifaldt A, Mordhorst LB and Sorbe B: Difference in outcome for cervical cancer patients treated with or without brachytherapy. Brachytherapy 16: 133-140, 2017.

25. Barraclough LH, Swindell R, Livsey JE, Hunter RD and Davidson SE: External beam boost for cancer of the cervix uteri when intracavitary therapy cannot be performed. Int J Radiat Oncol Biol Phys 71: 772-778, 2008.

26. Matsuura K, Okabe T, Fujita K, Tanimoto H, Akagi Y and Kagemoto M: Clinical results of external beam radiotherapy alone with a concomitant boost program or with conventional fractionation for cervical cancer patients who did not receive intracavitary brachytherapy. J Radiat Res 53: 900-905, 2012.

27. Ito K, Shimizuguchi $\mathrm{T}$ and Karasawa K: Clinical outcomes following conventional external beam radiotherapy boost in Japanese patients with cervical cancer who are ineligible for intracavitary brachytherapy. Jpn J Clin Oncol 49: 270-275, 2019

28. Park HC, Shimizu S, Yonesaka A, Tsuchiya K, Ebina Y, Taguchi H, Katoh N, Kinoshita R, Ishikawa M, Sakuragi N and Shirato H: High dose three-dimensional conformal boost using the real-time tumor tracking radiotherapy system in cervical cancer patients unable to receive intracavitary brachytherapy. Yonsei Med J 51: 93-99, 2010.

29. Saibishkumar EP, Patel FD, Sharma SC, Karunanidhi G, Sankar AS and Mallick I: Results of external-beam radiotherapy alone in invasive cancer of the uterine cervix: A retrospective analysis. Clin Oncol (R Coll Radiol) 18: 46-51, 2006.

30. Yoshida K, Yamazaki H, Kotsuma T, Takenaka T, Ueda MM, Miyake S, Tsujimoto Y, Masui K, Yoshioka Y, Sumida I, et al: Simulation analysis of optimized brachytherapy for uterine cervical cancer: Can we select the best brachytherapy modality depending on tumor size? Brachytherapy 15: 57-64, 2016.

31. Hong JH, Tsai CS, Lai CH, Chang TC, Wang CC, Chou HH, Lee SP and Hsueh S: Recurrent squamous cell carcinoma of cervix after definitive radiotherapy. Int J Radiat Oncol Biol Phys 60: 249-257, 2004. 